Revista Mexicana de Economía y Finanzas Nueva Época, Vol. 14 No. 1, (2019), pp. 113-128

DOI: http://dx.doi.org/10.21919/remef.v14i1.362

\title{
Efectos de corto y largo plazo de los programas de condonación de créditos fiscales en la recaudación del Impuesto al Valor Agregado
}

\author{
Javier Moreno ${ }^{1}$ \\ Universidad Anáhuac México Norte, México \\ Jaime H. Beltrán ${ }^{2}$ \\ Universidad Anáhuac México Norte, México \\ Leovardo Mata $^{3}$ \\ Universidad Anáhuac México Norte, México
}

(Primera recepción: 25/enero/2018, última recepción: 23/julio/2018, aceptado: 18/octubre/2018)

\section{Resumen}

El objetivo es determinar el impacto de corto y largo plazo de los programas de amnistía fiscal de 2007 y 2013 en la recaudación del IVA en México para el periodo 1980 - 2016. Para el análisis se utilizó un modelo ARIMA multivariado. Los resultados muestran que los programas de condonación de créditos fiscales no afectaron la recaudación del IVA en el largo plazo, el efecto predominante muestra un cambio de nivel con ajuste de corto plazo. Se recomienda no utilizar estos programas con fines recaudatorios, ya que no generan efectos en el largo plazo y tampoco benefician al cumplimiento voluntario. Para el análisis, fue necesario asegurar que se trabaja con series estacionarias y no correlacionadas. La originalidad radica en la metodología utilizada para determinar el impacto de los programas de amnistía fiscal promulgados por el gobierno de México para incrementar la recaudación pública. En consenso con la literatura, se confirma un efecto de corto plazo sobre el nivel de la recaudación del IVA y la ausencia de algún efecto de largo plazo. Clasificación JEL: E62

Palabras clave: amnistía, impuestos, recaudación, IVA, ARIMA multivariado

\section{Short and Long-Term Effects of Tax Credit Waiver Programs on Value Added Tax Collection}

\section{Abstract}

The objective of this work is to determine the short and long-term impact of the 2007 and 2013 fiscal amnesty programs on VAT collection in Mexico for the period of 1980-2016. A multivariate ARIMA model was used for the analysis. The results show that tax credit waiver programs did not affect VAT collection in the long term. The predominant effect shows a change of level with short-term adjustment. It is recommended to not use these programs for collection purposes, since they do not generate long-term effects and do not benefit voluntary compliance. For the analysis, it was necessary to ensure that stationary and non-correlated series are used. The originality of this lies in the methodology used to determine the impact of fiscal amnesty programs promoted by the Mexican government to increase tax collection. In consensus with the literature, a short-term effect is confirmed for VAT collection and the absence of any long-term effect.

\footnotetext{
${ }^{1}$ Candidato a Doctor en Gestión Estratégica y Políticas de Desarrollo por la Universidad Anáhuac México Norte.

${ }^{2}$ Profesor Investigador de la Universidad Anáhuac México Norte. Correo: jaime.beltrang@anahuac.mx. Tel. 4443332019

${ }^{3}$ Profesor Investigador de la Universidad Anáhuac México Norte.

*Sin fuente de financiamiento declarada para el desarrollo de la investigación
} 


\section{Abstract}

JEL Classification: E62

Keywords: Amnesty, taxes, collection, VAT, multivariate ARIMA

\section{Introducción}

La condonación de impuestos o créditos fiscales es una herramienta utilizada por los gobiernos con el objetivo de que los contribuyentes evasores corrijan su situación irregular ante la autoridad tributaria. A nivel internacional han destacado los casos de Irlanda y Colombia.

Uchitelle (1989) identificó que el Gobierno de Irlanda instrumentó en 1988 un programa de amnistía por diez meses, a través del cual condonó impuestos, intereses y recargos, determinó la no persecución de ningún contribuyente que se hubiese adherido al programa. Este programa fue acompañado con medidas estrictas de fiscalización, de tal forma que los contribuyentes percibieron la fuerza del Estado para hacer cumplir la Ley tributaria. Los resultados del programa alcanzaron una recaudación de 750 millones de dólares (2.5\% del PIB) y un incremento de la base gravable.

Uchitelle (1989) también estudió las características del programa de condonación de impuestos implementado en Colombia en 1987. Este programa determinó que las personas adheridas al programa de amnistía quedaban registradas en el padrón de contribuyentes y podrían ser auditadas en el futuro. El programa fue dirigido a los contribuyentes que no hubieran declarado ingresos en años anteriores a 1987, a quienes se les permitió regularizar su situación ante el fisco, sin ser sancionados o investigados en ese momento. De forma paralela, el Gobierno de Colombia realizó modificaciones al sistema tributario haciéndolo más estricto, con lo cual, el principal resultado alcanzado fue una recaudación de $0.3 \%$ del PIB en 1987.

En México, el Servicio de Administración Tributaria ha implementado dos programas de condonación de créditos fiscales, uno en 2007 y otro en 2013, ambos aprobados por el H. Congreso de la Unión. Entre sus alcances se encuentran diversas remisiones tales como la de contribuciones federales, cuotas compensatorias, multas por incumplimiento, obligaciones fiscales federales distintas a las obligaciones de pago, así como actualizaciones y recargos (LIF, 2007 y LIF 2013).

Uno de los resultados del programa de condonación de contribuciones federales de 2007 fue la recaudación de 14,598 millones de pesos y la condonación de 86,992 millones de pesos por concepto de adeudos fiscales.

Por su parte, el programa de condonación de 2013 registró una recaudación de 40,335 millones de pesos y una condonación de 166,479 millones de pesos por concepto de créditos fiscales. De acuerdo a las cifras disponibles que la Secretaría de Hacienda y Crédito Público tiene por tipo de impuesto y monto total cobrado, el IVA representó el $10 \%$, solo por debajo de la cifra cobrada por concepto del Impuesto Sobre la Renta. Aunado a esto, el monto total condonado, también relacionado con el IVA, representó un $3 \%$. En conjunto ambos montos sumaron 8,567 millones de pesos.

Siendo el IVA la segunda fuente de ingresos de la Hacienda mexicana, y al no existir una evaluación de los efectos en la recaudación del IVA de los programas de condonación de contribuciones federales, se decide estudiar los efectos de corto y largo plazo de los programas de condonación sobre la recaudación del IVA, mediante el análisis de series de tiempo y utilizando el enfoque propuesto por Alm y Beck (1993), a través del cual mediante un método ARIMA multivariado se buscará determinar el impacto (estadísticamente significativo) de los programas de amnistía en la recaudación del IVA y si dichos efectos tuvieron efectos de corto o largo plazo. 
Revista Mexicana de Economía y Finanzas Nueva Época, Vol. 14 No. 1, (2019), pp. 113-128

\section{Objetivo General}

El objetivo de este trabajo es medir el impacto de corto y largo plazo de los programas de condonación de créditos fiscales 2007 y 2013 en el nivel y la tendencia de la recaudación del IVA para el periodo 1980 - 2016.

\section{Revisión de literatura}

El fundamento teórico para el establecimiento de los diferentes impuestos y programas de amnistía se ubica en el campo teórico de las finanzas públicas; y el fundamento jurídico para el establecimiento de los programas de amnistía es la norma jurídica que proviene de la norma fundante que otorga validez al sistema jurídico que opera en México y que le otorga al H. Congreso de la Unión la facultad para establecer dichos programas de amnistía fiscal. Con estos fundamentos, la revisión de literatura permite identificar las fortalezas o debilidades en cuanto a la utilización de programas de amnistía como instrumento para incrementar la recaudación en los diferentes países.

Uchitelle (1989) estableció que los programas de amnistía fiscal tienen como objetivo primordial que los contribuyentes rectifiquen y se pongan al corriente con sus obligaciones. Por su parte, Fisher (2005) definió que los principales objetivos de un programa de amnistía son: la recaudación de impuestos en el corto plazo; el incremento de la recaudación en el largo plazo, a través de influir en el comportamiento del contribuyente para que los evasores cumplan con sus obligaciones fiscales adecuadamente; y la recopilación de información de los contribuyentes participantes, para ser utilizada en la vigilancia futura del cumplimiento de los contribuyentes.

Duhaime (2007) coincidió con Fisher (2005) respecto a la definición de amnistía fiscal, al establecer que ésta es una facilidad otorgada al contribuyente para corregir sus errores u omisiones en materia de impuestos causados en el pasado, sin ninguna penalización o persecución por parte de la autoridad tributaria. Mientras que Mikesell (1986) concluyó que el efecto más importante de un programa de amnistía se produce cuando el contribuyente percibe que las formas de evadir impuestos no se erradican después de la vigencia del programa; es decir, el contribuyente percibe que puede seguir evadiendo impuestos en el largo plazo. Esto significa que el programa de amnistía no fue acompañado con acciones de fiscalización y por tanto se generan incentivos a evadir.

Adicionalmente, Alm y Beck (1990) infirieron que el impacto de las amnistías sobre la recaudación de impuestos es ambiguo debido a que pueden tener un efecto positivo sobre la recaudación. Lo anterior sucede si los contribuyentes perciben que pagar impuestos es la norma y que en el futuro habrá medidas de fiscalización más severas a las existentes antes de la amnistía. También puede haber una consecuencia negativa al disminuir el cumplimiento voluntario cuando los contribuyentes perciben la existencia de otra amnistía futura con aplicación laxa.

Por otro lado, Andreoni (1991) afirmó que la variable más relevante para determinar si la recaudación de un impuesto aumentará o disminuirá como resultado de un programa de amnistía, es el nivel de evasión existente y no el cambio causado por la amnistía misma. Es decir, cuando la evasión inicial es muy grande, la amnistía puede aumentar los ingresos fiscales. Mientras que Dubin, Graetz y Wilde (1992) discurrieron que cuando se utilizan los programas de amnistía, se debe evaluar el rendimiento potencial de la amnistía más que la situación fiscal que presenta el Estado al momento de establecer la condonación de impuestos. Identificaron que cuando se mantiene una tasa significativa de ejecución de auditorías durante un periodo, la probabilidad de establecer una amnistía disminuye en casi un 25

Asimismo, Alm y Beck (1993) demostraron mediante mínimos cuadrados ordinarios, modelos de tendencia, modelos autoregresivos univariados con medias móviles y autoregresivos multivariados con medias móviles, que los programas de amnistía no tienen 
REMEF (The Mexican Journal of Economics and Finance)

Efectos de corto y largo plazo de los programas de condonación de

impacto en el largo plazo en el nivel y en la tendencia de la recaudación de impuestos. Los autores afirmaron que un programa típico de amnistía difícilmente generará recaudación nueva de impuestos y concluyeron que tampoco se tiene efecto sobre el cumplimiento voluntario de los contribuyentes.

Estos modelos matemáticos utilizaron series de tiempo, con el objetivo de pronosticar en el largo plazo el comportamiento de la recaudación del impuesto al ingreso con base en información histórica. De acuerdo con Zellner y Palm (1974), un modelo fundamentado en análisis de series de tiempo puede ser visto como una propuesta simplificada de un modelo estructural de mayor complejidad.

Alm y Beck (1993) también dedujeron que una forma de incrementar la recaudación después del establecimiento de un programa de amnistía, es el ejercicio por parte de la autoridad tributaria de las facultades de fiscalización, además de ofrecer mejores servicios al contribuyente para mejorar el cumplimiento de las obligaciones. Por su parte, Luitel y Sobel (2005) afirmaron que los programas de amnistía incrementan la recaudación en el corto y largo plazo, a través de captar nuevamente a los no declarantes del fisco. Sin embargo, estos últimos también señalaron que cuando se utilizan programas de amnistía en más de una ocasión, estos generan muy poca recaudación de corto plazo, en comparación con los programas antecesores y tienden a disminuir la recaudación en el largo plazo, ya que se desincentiva el cumplimiento voluntario de los contribuyentes.

Le Borgne (2006) confirmó que las amnistías fiscales se utilizan con mayor frecuencia durante periodos de estrés fiscal y cuando factores políticos influyen en la decisión de establecer programas de amnistía fiscal. En particular, demostró empíricamente que los políticos perciben a las amnistías fiscales como una fuente de ingresos adicional (en lugar de una alternativa de aumento de impuestos). También proporcionó evidencias que demuestran que cuando se rompe la equidad horizontal, las amnistías se pueden percibir como injustas; es decir, demostró que existe una correlación significativa entre la pérdida de elecciones y la introducción de una amnistía fiscal en periodos de campaña.

Los investigadores Bose y Jetter (2010) ratificaron que el éxito de un programa de amnistía está determinado por los cambios económicos, tales como la liberalización de la economía o el progreso tecnológico. Identificaron que cuando la autoridad tributaria solo tiene una visión recaudadora, no debería utilizar programas de amnistía, tendría que buscar una mejoría en la economía para que los diversos agentes se desarrollen dentro del sector formal. Analizaron que cuando el marco legal otorga incentivos a los evasores de impuestos para que se vuelvan honestos, el programa puede alcanzar resultados positivos.

Otras conclusiones relevantes fueron las de Mikesell et al (2012), quien afirmó que el propósito fundamental de los programas de amnistía ha cambiado de mejorar la administración tributaria a incrementar la recaudación de impuestos. Los resultados que obtuvieron revelan que la mayoría de las características de los programas de amnistía que tienden a aumentar la recaudación impositiva entran en conflicto o socavan el objetivo de mejorar el sistema de administración tributaria.

Bayer et al (2014) diseñaron un modelo que considera a los programas de amnistía de forma endógena; es decir, como resultado de decisiones estratégicas entre muchos contribuyentes que descuentan los pagos futuros del castigo y un gobierno que intercambia los costos y beneficios de dichos programas. Concluyeron que la probabilidad de establecer amnistías está principalmente impulsada por los requisitos que se establecen por parte del gobierno y por las expectativas de los contribuyentes acerca de posibles amnistías futuras.

Asimismo, Marchese (2014) concluyó que las amnistías pueden ser utilizadas como un mecanismo de discriminación para mejorar la eficiencia o la equidad del sistema tributario; sin embargo, este fin se alcanza cuando se tiene la percepción de que el gobierno hará cumplir la Ley tributaria. Si la credibilidad en el gobierno es mínima, la amnistía puede disminuir la recaudación futura al romperse la relación implícita entre los contribuyentes y el Estado, reduciendo la motivación de los contribuyentes para cumplir con 
Revista Mexicana de Economía y Finanzas Nueva Época, Vol. 14 No. 1, (2019), pp. 113-128

sus obligaciones fiscales.

Mouloud (2015) por su parte, analizó la relación entre los programas de amnistía fiscal y la economía informal, determinó que la informalidad es un fenómeno que afecta el crecimiento económico y la recaudación de impuestos; situación que se puede mitigar a través de la utilización de programas de amnistía, sin embargo concluyó que la amnistía deberá estar acompañada de algunas medidas, tales como: la eficiencia de la administración tributaria, un gobierno creíble y efectivo, incentivos fiscales que promuevan la inversión en el sector formal, menores tasas impositivas para la amnistía y servicios al contribuyente que faciliten el cumplimiento de sus obligaciones.

Sanchez (2017) estudió el efecto de las amnistías fiscales en la recaudación de impuestos en el largo plazo cuando el gobierno usa estos programas como fuente habitual de ingresos. Los principales resultados fueron: que las amnistías no afectan los ingresos de largo plazo y el aumento que se da en la recaudación es temporal y de corto plazo, aceleran la recaudación, pero no aumentan la cantidad recaudada. Concluyó que los programas de amnistía han sido utilizados como mecanismo para obtener ingresos en el corto plazo y para evitar reformas estructurales a los sistemas impositivos.

Finalmente, Sa'adah (2018), examinó la relación entre los programas de amnistía fiscal y la inversión y consideró que la amnistía fiscal se puede convertir en un instrumento de política económica para atraer ciudadanos que busquen repatriar e invertir en un país. Concluyó que la amnistía fiscal es teóricamente capaz de incentivar la inversión nacional, sin embargo, ésta no puede garantizar el incremento de la inversión de forma aislada, debe ser acompañada de otras políticas públicas que respalden la inversión, tales como garantizar certidumbre en las políticas impositivas y ofrecer seguridad al inversionista.

Con base en la revisión de la literatura, se concluye que los programas de amnistía con fines recaudatorios, tienen efectos negativos en la propia recaudación; existen factores que pueden revertir el efecto negativo, tales como, la aplicación estricta de las facultades de fiscalización posteriores al programa de amnistía y a la generación de percepción de riesgo en los contribuyentes de que el Estado no es débil en la aplicación de las leyes fiscales.

La evaluación del impacto de los programas de amnistía de 2007 y 2013 sobre la recaudación del IVA durante el periodo de 1980 a 2016, se realizará mediante los métodos utilizados por Alm y Beck (1993) a través del análisis de series de tiempo y la utilización del método ARIMA multivariado, con el objeto de confirmar si dichos programas tuvieron algún efecto sobre la recaudación del IVA en el corto o largo plazo para el caso de México.

\section{Construcción del indicador de amnistía}

El análisis del impacto de los programas de amnistía fiscal de 2007 y 2013 en la recaudación del Impuesto al Valor Agregado en México, se realiza a partir de la revisión de los dos programas de condonación de adeudos al fisco con características similares respecto a los beneficios otorgados a los contribuyentes no cumplidos para regularizar su situación fiscal.

Estos programas fueron establecidos por el Gobierno de México en la Ley de Ingresos de la Federación aprobada por el H. Congreso de la Unión, para los ejercicios fiscales de 2007 y 2013. La vigencia de los dos programas fue: para el caso de 2007 con una duración de un año y tres meses, del 1 de enero de 2007 al 31 de marzo de 2008; para el programa de 2013, con una duración de tres meses y 10 días, del 19 de febrero de 2013 al 31 de mayo del mismo año.

\subsection{Programas de condonación de adeudos}

Las principales características de los dos programas de amnistía los hacen comparables, en ambos casos se condonan contribuciones federales y fueron aprobados por el H. Congreso de la Unión. Los alcances y beneficios de cada uno de los programas de amnistía se presentan a continuación. 
REMEF (The Mexican Journal of Economics and Finance)

Efectos de corto y largo plazo de los programas de condonación de

\subsubsection{Programa de condonación de adeudos 2007}

El programa de condonación de contribuciones federales de 2007, otorgó los siguientes beneficios (SHCP, 2013):

1. Para el ejercicio de 2007:

a. $50 \%$ en multas por incumplimiento de las obligaciones fiscales distintas a las de pago;

2. Para los ejercicios de 2003 a 2005 :

a. $100 \%$ en recargos y multas,

b. $0 \%$ en contribuciones y cuotas compensatorias;

3. Para el ejercicio de 2002 o anteriores:

a. $100 \%$ si el contribuyente tuvo alguna revisión en los ejercicios 2004, 2005 y 2006 y el resultado fue sin observaciones y en caso de que hubiera observaciones el contribuyente las hubiere pagado y se encuentre al corriente,

b. $80 \%$ en multas por incumplimiento de las obligaciones fiscales distintas a las de pago,

c. $100 \%$ en recargos, multas y gastos de ejecución,

d. $80 \%$ en contribuciones y cuotas compensatorias.

La vigencia del programa fue de un año tres meses y los resultados fueron los siguientes:

- 14,598 millones de pesos recuperados por adeudos fiscales.

- 86,992 millones de pesos condonados.

\subsubsection{Programa de condonación de adeudos 2013}

El programa de condonación de contribuciones federales de 2013, otorgó los siguientes beneficios (SHCP, 2013):

1. Para el ejercicio de 2013:

a. $60 \%$ en multas por incumplimiento de las obligaciones fiscales distintas a las de pago;

2. Para los ejercicios de 2007 a 2012:

a. $100 \%$ en recargos y multas,

b. $0 \%$ en contribuciones y cuotas compensatorias,

c. $100 \%$ en multas por incumplimiento de las obligaciones fiscales distintas a las de pago.

3. Para el ejercicio de 2006 o anteriores:

a. $100 \%$ si el contribuyente tuvo alguna revisión en los ejercicios 2009, 2010 y 2011 y el resultado fue sin observaciones y en caso de que hubiera observaciones el contribuyente las hubiere pagado y se encuentre al corriente,

b. $80 \%$ en multas por incumplimiento de las obligaciones fiscales distintas a las de pago,

c. $100 \%$ en recargos, multas y gastos de ejecución, 
d. $80 \%$ en contribuciones y cuotas compensatorias.

La vigencia de este programa fue de tres meses y diez días y los resultados fueron los siguientes:

- 40,335 millones de pesos recuperados por adeudos fiscales.

- 166,479 millones de pesos condonados.

\subsection{Estimación del impacto mediante un modelo ARIMA}

El análisis de los efectos de los programas de condonación de contribuciones federales de 2007 y 2013 sobre la recaudación del IVA se construyó bajo la metodología propuesta por Alm y Beck (1993), en la cual, mediante el análisis de series de tiempo y con el método denominado Autoregresivo Integrado de Medias Móviles (ARIMA) multivariado se examinan los efectos en el corto y largo plazo de los programas de condonación. Para ello se utilizó el método ARIMA que permite estimar el comportamiento futuro de una variable a partir de los propios datos observados en el pasado. Estos modelos no requieren ser derivados de teoría económica alguna, dado que se calculan con los rezagos de la misma variable.

El método establece que se debe trabajar con series de tiempo estacionarias o en su caso, series de tiempo no estacionarias, pero que después de una o más diferenciaciones se conviertan en series de tiempo estacionarias. La razón de trabajar con series de tiempo estacionarias, de acuerdo a los autores de la metodología de $\mathrm{BJ}^{4}$, se explica así:

El objetivo de BJ[Box-Jenkins] es identificar y estimar un modelo estadístico que se interprete como generador de los datos muestrales. Si se va a pronosticar con este modelo estimado, debe suponerse que sus características son constantes a través del tiempo y, en particular, en periodos futuros. La razón para requerir datos estacionarios es que todo modelo que se infiera a partir de estos datos pueda interpretarse como estacionario o estable en sí mismo, y proporcione, por consiguiente, una base válida para pronosticar (Pokorny, 1987)

Las características de un modelo ARIMA son las siguientes (Montgomery, 2005): es estacionario, invertible, los parámetros estimados son de alta calidad, es parsimonioso ya que tiene un número mínimo de parámetros, sus residuos son un proceso de ruido blanco, ajusta bien los datos (R2) y tiene un error de predicción relativamente bajo.

Cuando se realiza una buena identificación, estimación y especificación del modelo ARIMA, este proporciona un excelente ajuste de los datos existentes y ofrece buenas predicciones de comportamiento futuro.

En la figura 1 se muestra la serie de recaudación del IVA para el periodo de enero de 1980 a diciembre de 2016, se identifica el periodo de vigencia de los dos programas de amnistía fiscal, con la finalidad de mostrar la unidad de análisis en el modelo ARIMA ante las dos intervenciones definidas como los programas de condonación de adeudos de 2007 y de 2013.

\footnotetext{
${ }^{4}$ G.P.E. Box and GM Jengins, "Time Series Analysis: Forecasting and Control".
} 
Figura 1. Recaudación del Impuesto al Valor Agregado 1980-2016

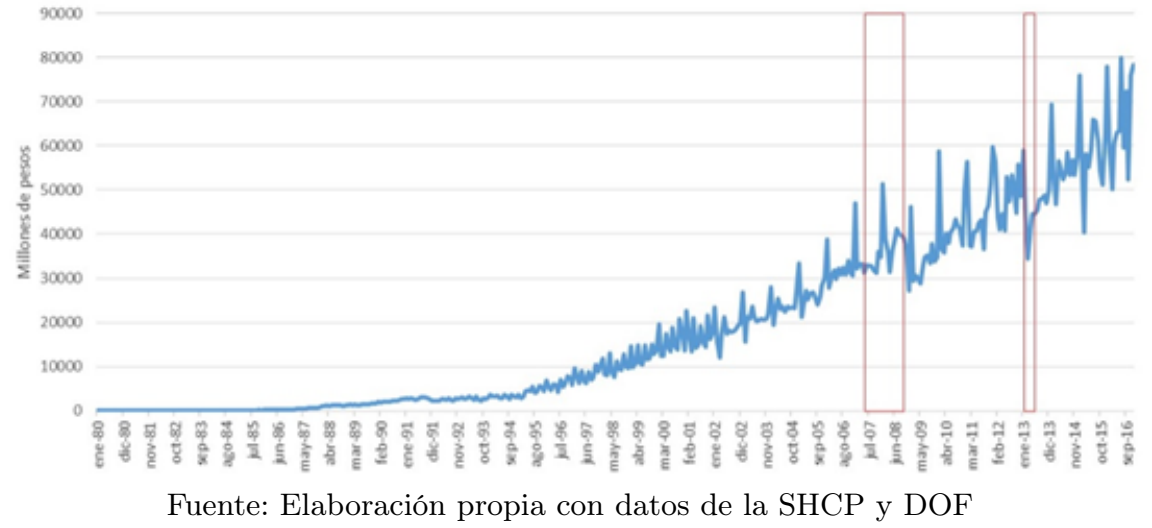

Con base en los procedimientos establecidos para un modelo ARIMA, se descompuso el logaritmo natural de la serie original de la recaudación del IVA para identificar y validar su estacionalidad y su tendencia y se identificó lo siguiente.

La recaudación del IVA presenta una tendencia creciente a lo largo del tiempo, con una estacionalidad bien definida y homogénea y un comportamiento errático aleatorio en años posteriores al año 2000.

En la figura 2 se observa la serie logarítmica y los tres elementos (tendencia, estacionalidad y aleatoriedad) en los que se descompone la serie original de la recaudación del IVA.

Figura 2. Tendencia, estacionalidad y aleatoriedad de la recaudación del IVA

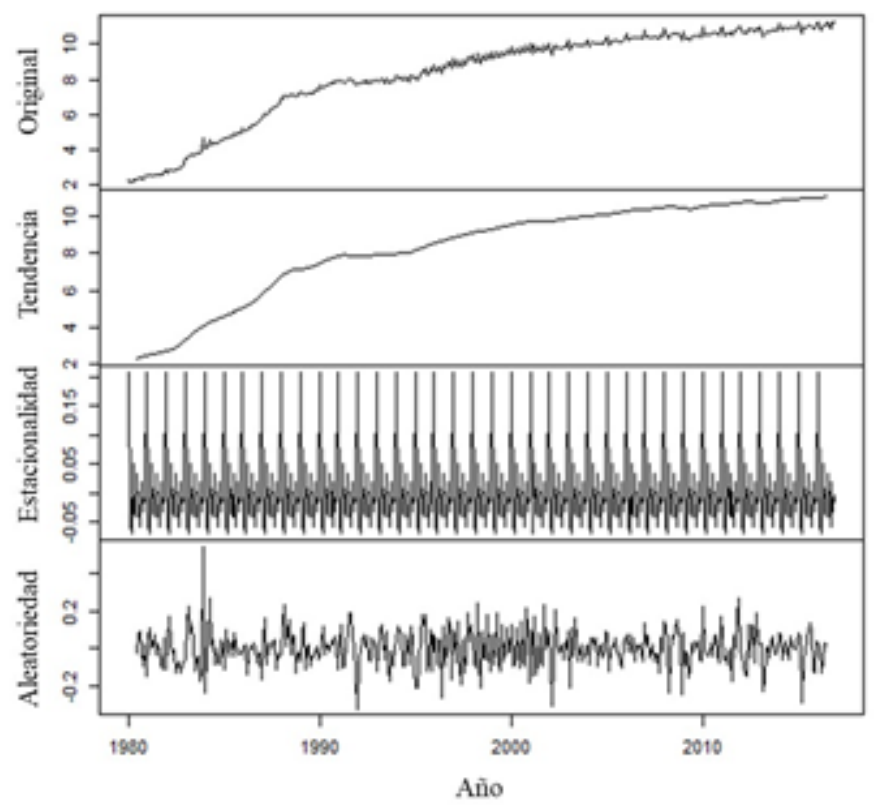

Fuente: Elaboración propia con datos de la SHCP y DOF

La metodología establece que se debe trabajar con series estacionarias para que la estimación de los parámetros de mejor ajuste en un modelo ARIMA sean de buena calidad; por lo tanto, se segmentó la serie de tiempo entre pre y post intervención, a fin de obtener un modelo que tenga presente la tendencia de la serie antes del programa de amnistía, el cual fungirá como muestra de control, para determinar el impacto de los choques a la serie de recaudación del IVA. 
Para estimar periodos futuros de acuerdo al comportamiento de la serie previa a la intervención, basándose en la función de autocorrelación para determinar el componente MA(q) y la función de autocorrelación parcial para el componente $\operatorname{AR}(p)$ y tomando en cuenta la estacionalidad de la serie, se analizó una serie de modelos, determinando de acuerdo al criterio de Akaike (-487.87) y al criterio de información bayesiano (-456.06), que el modelo ARIMA $(5,2,0) \times(2,0,0)(12)$ era el de mejor ajuste para los fines de esta investigación.

Un ARIMA $(5,2,0) \times(2,0,0)(12)$, cuya ecuación está definida como:

$$
\begin{gathered}
\varphi_{5}(B) \Phi_{2}\left(B^{12}\right) \nabla^{2} y_{t}=c+\varepsilon_{t} \\
\left(1-\varphi_{5} B\right)\left(1-\Phi_{2} B^{12}\right)\left(1-B^{2}\right) y_{t}=c+\varepsilon_{t}
\end{gathered}
$$

En donde:

$y_{t}=$ Recaudacin del IVA

$\varphi=$ Valor de los coeficientes AR

$\Phi=$ Valor de los coeficientes SAR

$\mathrm{B}=$ operador de rezagos

implica que el modelo para el análisis de la recaudación del IVA contiene 5 términos autorregresivos, lo que significa que el dato en t depende de sus 5 últimos resultados; una diferenciación de orden 2 a fin de eliminar la tendencia y convertir la serie en estacionaria y; 2 términos autorregresivos estacionales (SAR) que indican una estacionalidad anual. Los resultados que arroja el modelo con mejor ajuste, se explicarán en el siguiente apartado.

Este tipo de modelos, presentan algunas desventajas, son modelos complejos, que utilizados en datos que presentan correlación pueden arrojar estimaciones no consistentes; razón por la cual, se revisó a profundidad los diagramas de autocorrelación total y parcial, para cuidar que las estimaciones sean consistentes.

\section{Resultados de la evaluación del impacto de los programas de amnistía}

Para identificar los efectos de los programas de condonación de adeudos, se analizaron los efectos de manera individual y posteriormente por contraste se identificaron las coincidencias y las divergencias en los resultados de cada uno de los programas instrumentados por el Gobierno de México, en cuanto a la condonación de adeudos fiscales en materia del IVA.

\subsection{Programa de condonación de adeudos de contribuciones fede- rales 2007}

Mediante el modelo propuesto en el apartado 4.2 se analizó el periodo previo a la intervención del programa (enero de 1980 a diciembre de 2006) para determinar los componentes del modelo ARIMA que se utilizarán en la estimación del tipo y la magnitud de la intervención. En el cuadro 1 se muestran los valores de los coeficientes que arroja el modelo.

Cuadro 1. Valor de los parámetros del modelo ARIMA $(5,2,0)(2,0,0)$

\begin{tabular}{|c|c|c|c|c|c|c|c|}
\hline \multicolumn{7}{|c|}{ Modelo ARIMA (5,2,0)(2,0,0)(12) sin intervención } \\
\hline & ar1 & ar2 & ar3 & ar4 & ar5 & sar1 & sar2 \\
\hline Coeficientes & -1.2942 & -1.3084 & -0.9592 & -0.5731 & -0.3624 & 0.3304 & 0.2027 \\
\hline Error estándar & 0.0529 & 0.0873 & 0.098 & 0.0849 & 0.0546 & 0.0586 & 0.0582 \\
\hline \multicolumn{7}{|c|}{ Fuente: Estimación propia. }
\end{tabular}

La prueba de significancia " $\mathrm{z}$ " permite afirmar que el (p-value) del modelo para el periodo previo a la intervención del programa de condonación de adeudos de 2007 es significativo. La prueba de significancia se da al $99 \%$. En el cuadro 2 se muestran los resultados de la prueba " $\mathrm{z}$ ". 
REMEF (The Mexican Journal of Economics and Finance) Efectos de corto y largo plazo de los programas de condonación de

Cuadro 2. Prueba de significancia para el modelo ARIMA $(5,2,0)(2,0,0)$ Prueba z para los coeficientes del modelo ARIMA sin intervención

\begin{tabular}{|c|c|c|c|c|c|}
\hline & $\begin{array}{c}\text { Valor } \\
\text { coeficiente }\end{array}$ & $\begin{array}{c}\text { Error } \\
\text { estándar }\end{array}$ & Valor de $\mathrm{z}$ & $\operatorname{Pr}(>|z|)$ & Significancia \\
\hline ar1 & -1.2942 & 0.05287 & -24.4767 & $<2.2 \mathrm{E}-16$ & *** \\
\hline ar2 & -1.3084 & 0.08732 & -14.9823 & $<2.2 \mathrm{E}-16$ & *** \\
\hline ar3 & -0.9592 & 0.09797 & -9.7901 & $<2.2 \mathrm{E}-16$ & *** \\
\hline ar4 & -0.5731 & 0.08485 & -6.7546 & $1.43 \mathrm{E}-11$ & *** \\
\hline ar5 & -0.3624 & 0.05459 & -6.6386 & $3.17 \mathrm{E}-11$ & *** \\
\hline sar1 & 0.3304 & 0.05859 & 5.6377 & $1.72 \mathrm{E}-08$ & *** \\
\hline sar2 & 0.2027 & 0.05821 & 3.4817 & 0.0004982 & *** \\
\hline
\end{tabular}

Fuente: Estimación propia.

Por contraste, el modelo proporcionó resultados no significativos al simular la intervención en enero de 2007, que considera la vigencia del programa de condonación de adeudos de 2007.

Esto se confirma al observar los valores de los parámetros Jan07* que muestran el efecto de la intervención en la recaudación y que no son significativos al mismo nivel de confianza. En el cuadro 3 se muestran los valores de los parámetros estimados para la intervención del programa de condonación de adeudos.

Cuadro 3. Valor de los parámetros del modelo ARIMA $(5,2,0)(2,0,0)$ con

intervención en enero de 2007

\begin{tabular}{|c|c|c|c|c|c|c|c|c|c|c|}
\hline \multicolumn{10}{|c|}{ Modelo ARIMA } & $(\mathbf{5 , 2 , 0})(\mathbf{2 , 0 , 0}(\mathbf{1 2})$ con intervención \\
\hline & ar1 & ar2 & ar3 & ar4 & ar5 & sar1 & sar2 & Jan07a-MA0 & Jan07b-AR1 & Jan07b-MA0 \\
\hline Coeficientes & -1.2988 & -1.2633 & -0.9587 & -0.585 & -0.3353 & 0.4204 & 0.2419 & -0.0059 & 0.766 & 0.1069 \\
\hline Error estándar & 0.0452 & 0.0729 & 0.0823 & 0.0725 & 0.0468 & 0.0477 & 0.048 & 0.1391 & 0.4281 & 0.1356 \\
\hline
\end{tabular}

Sigma ${ }^{2}$ estimado0,01601 : loglikelihood $=282,36$, aic $=-544,71$

Fuente: Estimación propia.

La prueba "z" permite afirmar que la estimación de los parámetros con intervención del programa de condonación de adeudos de enero de 2007, no es significativa. En el cuadro 4 se muestran los resultados de la prueba que permite validar la significancia de la estimación.

Cuadro 4. Prueba de significancia para el modelo ARIMA $(5,2,0)(2,0,0)$ con intervención del programa de condonación de contribuciones 2007

\begin{tabular}{|c|c|c|c|c|c|}
\hline \multicolumn{5}{|c|}{ Prueba z para los coeficientes del modelo ARIMA con intervención } \\
\hline & Valor coeficiente & Error estándar & Valor de $\mathbf{z}$ & \multicolumn{1}{|c|}{$\operatorname{Pr}(>|\mathbf{z}|)$} & Significancia \\
\hline ar1 & -1.2988 & 0.0452 & -28.7571 & $<2.2 \mathrm{E}-16$ & $* * *$ \\
\hline ar2 & -1.2633 & 0.0729 & -17.327 & $<2.2 \mathrm{E}-16$ & $* * *$ \\
\hline ar3 & -0.9587 & 0.0823 & -11.6435 & $<2.2 \mathrm{E}-16$ & $* * *$ \\
\hline ar4 & -0.585 & 0.0725 & -8.0717 & $6.94 \mathrm{E}-16$ & $* * *$ \\
\hline ar5 & -0.3353 & 0.0468 & -7.1641 & $7.83 \mathrm{E}-13$ & $* * *$ \\
\hline sar1 & 0.4204 & 0.0477 & 8.8132 & $<2.2 \mathrm{E}-16$ & $* * *$ \\
\hline sar2 & 0.2419 & 0.048 & 5.042 & $4.61 \mathrm{E}-07$ & $* * *$ \\
\hline Jan07a-MA0 & -0.0059 & 0.1391 & -0.0424 & 0.9662 & \\
\hline Jan07b-AR1 & 0.766 & 0.4281 & 1.7895 & 0.0735 & \\
\hline Jan07b-MA0 & 0.1069 & 0.1356 & 0.7886 & 0.4304 & " \\
\hline
\end{tabular}

Fuente: Estimación propia.

A partir de los resultados del modelo con intervención, se optimizó la omega de la ecuación de transferencia ${ }^{5}$ para determinar la persistencia de la intervención en los datos.

\footnotetext{
${ }^{5}$ Se deberá entender por ecuación de transferencia al polinomio de la intervención.
} 
Se puede inferir a partir de esta optimización que la intervención que se realizó al modelo generó un cambio positivo, pero de corto plazo.

En la figura 3 se observa este efecto en el repunte que se muestra alrededor del valor de la omega de 0.8 , punto en donde se presenta el valor mínimo local del criterio de Akaike.

Figura 3. Criterio de Akaike para los diferentes valores de omega

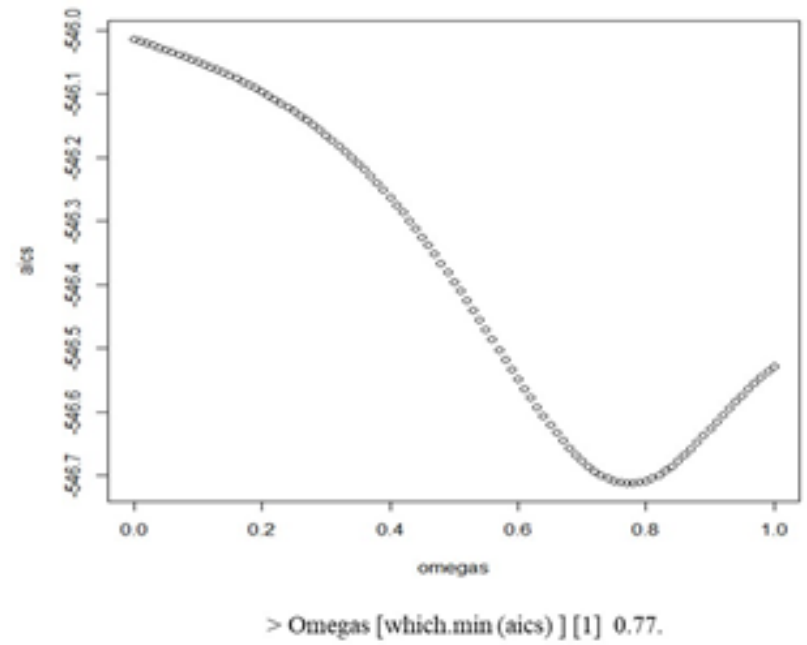

Fuente: estimación propia con datos de SHCP y DOF.

Para corroborar este resultado, se realizó la diferencia entre los datos y la estimación dado el modelo previo a la intervención, esta comparación permite afirmar que el impacto fue transitorio y de corto plazo.

En la figura 4 se muestra el efecto total de la intervención causado por el programa de condonación de adeudos fiscales de 2007: se observa una reacción significativa al inicio del programa de amnistía, pero se diluye con el paso del tiempo, esto significa que la recaudación del impuesto regresa a su comportamiento usual, sin mostrar un cambio de largo plazo provocado por la instrumentación del programa de condonación de adeudos.

Figura 4. Efecto total de la intervención del programa de condonación de adeudos 2007.

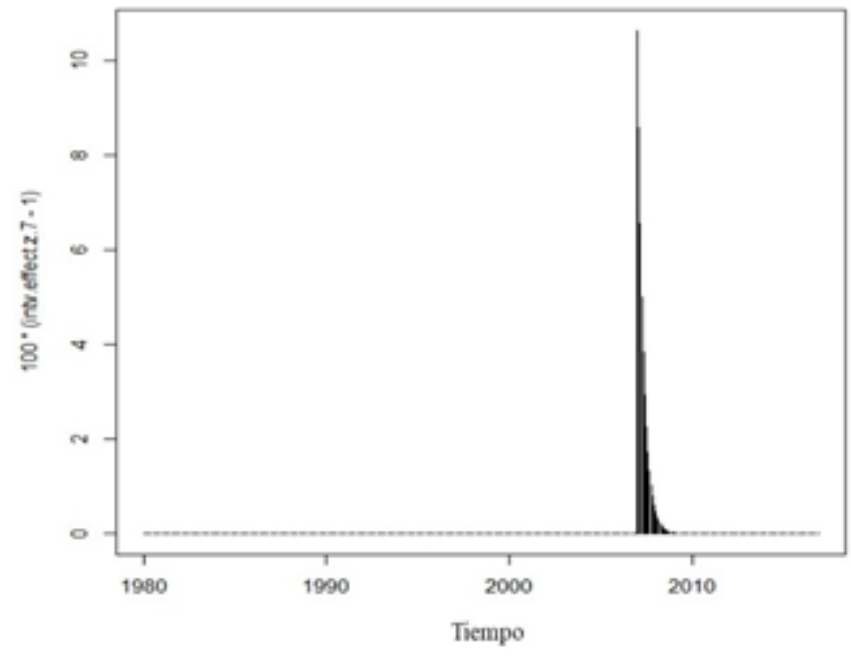

Fuente: estimación propia con datos de SHCP y DOF. 


\subsection{Programa de condonación de adeudos de contribuciones fede- rales 2013}

Para el análisis de los efectos del programa de condonación de adeudos de 2013, se procedió bajo los mismos criterios que se utilizaron para el programa de condonación de adeudos de 2007; es decir, se utilizó el mismo modelo propuesto en el apartado 4.2 en dos periodos distintos, uno previo a la entrada en vigor del programa de 2013 (enero de 1980 a diciembre de 2012) y otro a partir de 2013 para incorporar la vigencia del programa de condonación de contribuciones federales.

Para el periodo previo a la entrada en vigor del programa de condonación de contribuciones federales, se obtuvieron los siguientes resultados (ver apéndice A):

a. El modelo previo a la intervención es consistente con el modelo presentado para el programa de condonación de 2007, en su periodo previo a la intervención.

b. Los coeficientes son significativos al $99 \%$ y los residuales cumplen los supuestos, dando certeza al modelo.

Sin embargo, la simulación de la intervención del programa de condonación en enero de 2013, arrojó los siguientes resultados (ver apéndice A):

a. Los coeficientes son no significativos, cumpliendo lo esperado.

$\mathrm{Al}$ optimizar la omega en la ecuación de transferencia para determinar la persistencia de la intervención en los datos se obtuvo que el programa de condonación de contribuciones federales de 2013, generó un cambio positivo en la recaudación del IVA, pero de muy corto plazo.

En la figura 5 se observa un mínimo local en el criterio de Akaike dada la omega de transferencia cercana a cero (0.13); por lo tanto, se confirma que los efectos de la intervención fueron de corto plazo.

Figura 5. Criterio de Akaike para los diferentes valores de omega.

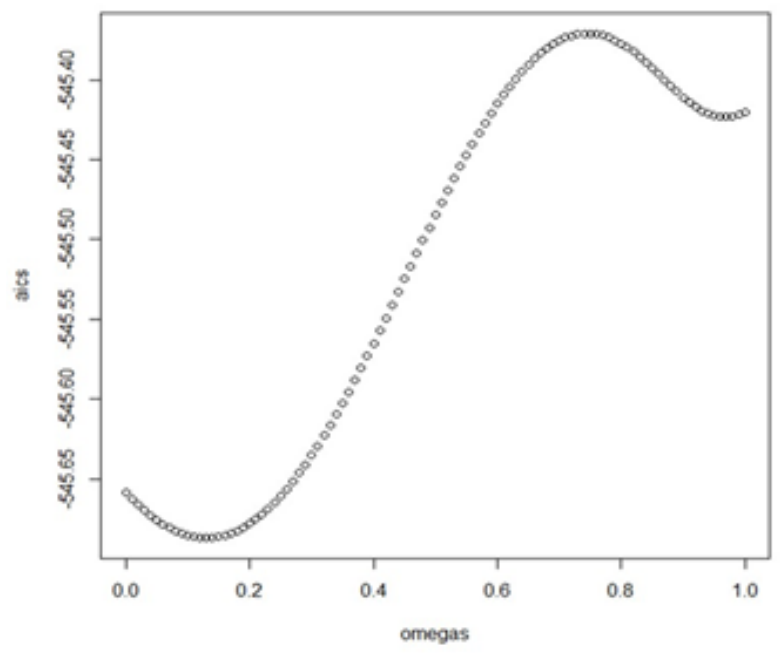

Fuente: estimación propia con datos de SHCP y DOF.

Para corroborar este resultado, se realizó la diferencia entre los datos y la estimación del modelo previo a la intervención, lo que permite afirmar que el impacto del programa de condonación de contribuciones del 2013 también fue transitorio y de corto plazo.

En la figura 6 se muestra el efecto total del programa de condonación de contribuciones federales 2013, se observa una reacción significativa al inicio del programa pero que se 
diluye con el paso del tiempo, esto significa que la recaudación del IVA regresa a su comportamiento usual en el corto plazo; hay que recordar que la vigencia de este programa fue de solo tres meses y diez días; por lo tanto, los efectos de este programa no muestran un cambio en la recaudación del IVA de largo plazo.

Figura 6. Efecto total de la intervención del programa de condonación de adeudos 2013

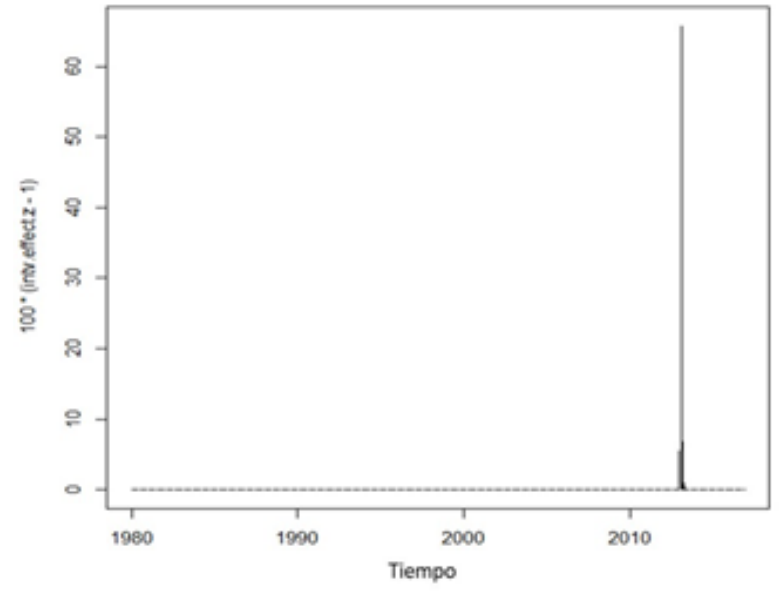

Fuente: estimación propia con datos de SHCP y DOF.

A partir del contraste de los datos observados de la recaudación del IVA con los datos estimados por el modelo propuesto en el apartado 4.2, se afirma que no hay un efecto significativo de largo plazo en la recaudación de IVA a partir del establecimiento de los programas de condonación de contribuciones federales establecidos por el Gobierno mexicano.

En la figura 7 se muestra la comparación de los datos observados de la recaudación del IVA, con los datos estimados por el modelo que considera la existencia de los programas de condonación de contribuciones federales. Se observa que no existen diferencias representativas que permitan identificar efectos significativos en el largo plazo de los programas de condonación de contribuciones.

Figura 7. Serie logarítmica de recaudación del IVA, periodo pre y post intervención,

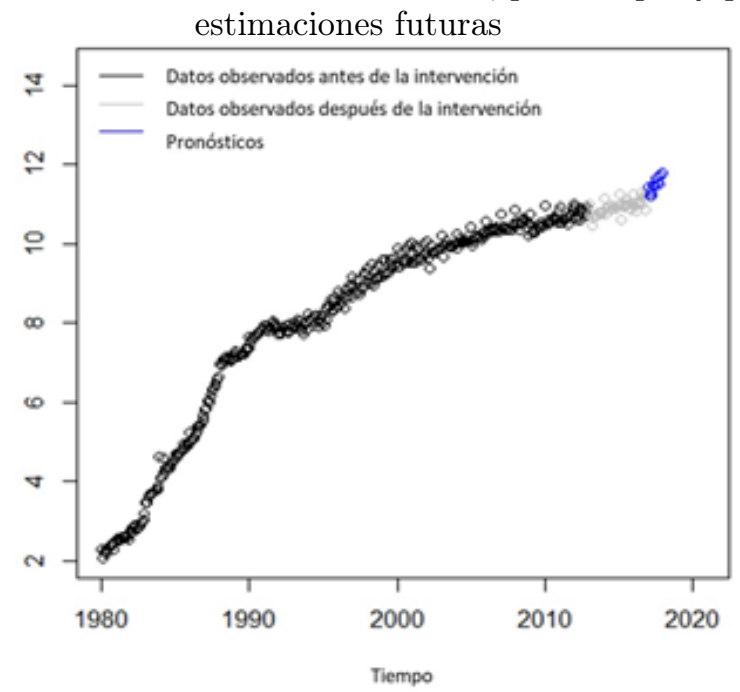

Fuente: estimación propia con datos de SHCP y DOF. 


\section{Conclusiones}

De acuerdo con Alm y Beck (1993), los programas de amnistía no tienen impacto en el largo plazo en el nivel y en la tendencia de la recaudación de impuestos. Estos autores afirmaron que un programa típico de amnistía difícilmente generará recaudación nueva de impuestos, y tampoco tendrá un efecto sobre el cumplimiento voluntario de los contribuyentes.

Los resultados de la aplicación del modelo ARIMA multivariado propuesto en el apartado 4.2 de este artículo, confirman que los dos programas de condonación de contribuciones federales utilizados por el Gobierno de México, no afectaron de manera permanente la recaudación del IVA y tampoco demostraron que se haya afectado el cumplimiento voluntario de los contribuyentes, ya que los efectos identificados se ajustaron a su trayectoria normal en el corto plazo.

El programa de condonación de contribuciones federales de 2007 presentó una mayor persistencia en la trayectoria de la recaudación del IVA en comparación con el programa de condonación de contribuciones federales de 2013. Este fenómeno se explica por la vigencia de los programas; el programa de 2007 tuvo una duración de un año y tres meses mientras que el programa de 2013 tuvo una vigencia de tres meses y 10 días.

Los resultados confirman el consenso que existe entre los investigadores respecto a los efectos de los programas de amnistía en la recaudación de impuestos. Los efectos se presentan en el corto plazo mediante un incremento transitorio de la recaudación y los efectos a largo plazo pueden ser incluso negativos sobre la propia recaudación. Este argumento se justifica mediante las cifras totales de recaudación y la condonación para cada uno de los programas; el programa de 2007 a pesar de haber tenido mayor vigencia, solo logró condonar 86,992 millones de pesos; mientras que el programa de 2013 con casi cinco veces menos tiempo de vigencia, se condonaron casi dos veces más de adeudos con el fisco.

De acuerdo a Le Borgne (2006), las amnistías fiscales se utilizan con mayor frecuencia durante periodos de estrés fiscal y cuando factores políticos influyen en la promulgación de éstos. Demostró empíricamente que los factores políticos pueden influir en el establecimiento de programas de amnistía cuando éstos consideran a estos programas como una fuente de ingresos, más que como una alternativa de aumento de recaudación; por lo tanto, establecer programas de condonación de impuestos con alta frecuencia o con enfoques de corte político, puede afectar la recaudación de la hacienda mexicana en el mediano y largo plazo.

Por lo anterior, se recomienda a las autoridades tributarias de los diferentes países no utilizar programas de amnistía con fines recaudatorios, ya que los efectos alcanzados a través de estos instrumentos de política fiscal, son transitorios y de corto plazo; además de que pueden disminuir los incentivos para llevar a cabo los cambios estructurales en el sistema tributario nacional. 


\section{Referencias}

Alm, J., Beck, W. (1990). Tax amnesties and Tax Revenues. Public Finance Review 1990.

Alm, J., Beck, W. (1993). Tax amnesties and compliance in the long run: a time series analysis. National Tax Journey, 46(1), 53-60.

Andreoni (1991). The desirability of a permanent tax amnesty. Journal of Public Economics 45, 143-159. North Holland.

Bayer R. et al (2014). The occurrence of Tax Amnesties: Theory and Evidence. SSRN Electronic Journal, 125, January 2014.

Bose, P. Jetter, M. (2010), A Tax Amnesty in the Context of a Developing Economy. Department of Economics, Fogelman College of Business and Economics, University of Memphis, Memphis, TN 38152, USA May 13, 2010.

Cámara de Diputados del H. Congreso de la Unión (2006). Ley de Ingresos de la Federación para el Ejercicio Fiscal 2007, LIF 2007. Diario Oficial de la Federación, 27 de diciembre de 2006.

Cámara de Diputados del H. Congreso de la Unión (2012). Ley de Ingresos de la Federación para el Ejercicio Fiscal 2013, LIF 2013. Diario Oficial de la Federación, 17 de diciembre de 2012.

Dubin, J., Graetz, M., Wilde, L. (1992). State Income Tax Amnesties: Causes. The Quarterly Journal of Economics 107 No. 3, August 1992. 1057-1070

Duhaime. (2007). Dictionary Law. Recuperado el 6 de octubre de 2015, de http://www.duhaime.org/ LegalDictionary.aspx

Fisher (2005). The Encyclopedia of Taxation Tax Policy online. Recuperado el 3 de mayo de 2017 en https://books.google.com.mx/books?id=XsGdBReOQSgC\&printsec=frontcover\&hl=es\#v=onepage\& $\mathrm{q} \& \mathrm{f}=\mathrm{false}$

Le Borgne (2006), E. Economic and Political Determinants of Tax Amnesties in the U.S. States. International Monetary Fund, Fiscal Affair Department, Working Paper 2006/06/222

Luitel, H., Sobel, R. (2005). The Revenue Impact of Repeated Tax Amnesties. Department of Economics. St. Cloud State University, october 22, 2005

Marchese, C. (2014). Tax Amnesties. International Programme in Institutions, Economics and Law IEL, paper in Comparative Analysis of Institutions, Economics and Law No. 17.

Mikesell, J. (1986). Amnesties for state tax evaders: the nature and response to recent programs. National tax journal, 4(39), 507-525.

Mikesell J. (2012). Fast Money? The contribution of State Tax Amnesties to Public Revenue Systems. National Tax Journal, 65(3), 529-562.

Montgomery, D. (2005). Introduction to statistical quality control (5 ed.). John Wiley Sons.

Mouloud, M. (2015). The tax amnesty program: as a tool to adjust the shadow economy; the international experiences. Global Advanced Research Journal of Economics, Accounting and Finance Vol. 3 (2) pp. 017-025, August, 2015

Pokorny, M. (1987). An introduction to econometrics. Nueva York: Basil Blackwell.

Sa'adah, N. (2018). Tax amnesty policy as an effort to improve state revenues and investment growth. IOP Conf. Series: Earth and Environmental Science 175 (2018), Faculty of Law, Diponegoro University, Semarang, Indonesia.

Sanchez, M. (2017). On the effects of repeated tax amnesties. Journal of Economics and Political Economy. Volume 4, Issue 3, September 2017.

SHCP. (2013). Secretaría de Hacienda y Crédito Público, Ponte al Corriente 2013.

Uchitelle, E. (1989). The effectiveness of Tax Amnesty Programs in Selected Countries. Federal Reserve Bank of New York, 48-53.

Zellner, A., Palm, F. (1974). Time series analysis and simultaneous equation econometric models. Journal of econometrics, 2.

\section{Apéndice A}

En el cuadro 1 de este apéndice se muestra el valor de los parámetros estimados para el programa de condonación de contribuciones federales de 2013, para el periodo previo a la vigencia del programa (enero de 1980 a diciembre de 2012).

Cuadro 1. Valor de los parámetros del modelo ARIMA $(5,2,0)(2,0,0)$, sin intervención en enero de 2013. 
REMEF (The Mexican Journal of Economics and Finance) Efectos de corto y largo plazo de los programas de condonación de créditos fiscales en la recaudación del Impuesto al Valor Agregado

\begin{tabular}{|c|c|c|c|c|c|c|c|}
\hline \multicolumn{7}{|c|}{ Modelo ARIMA (5,2,0)(2,0,0)(12) sin intervención } \\
\hline & ar1 & ar2 & ar3 & ar4 & ar5 & sar1 & sar2 \\
\hline Coeficientes & -1.2821 & -1.2586 & -0.9309 & -0.5601 & -0.3254 & 0.3963 & 0.2325 \\
\hline Error estándar & 0.0482 & 0.077 & 0.0868 & 0.0761 & 0.0492 & 0.0513 & 0.0507 \\
\hline
\end{tabular}

Sigmâ2 estimado 0.01627: $\log$ likelihood $=251.94, \mathrm{AIC}=-487.87, \mathrm{AICc}=-487.5, \mathrm{BIC}=-456.06$

Fuente: Estimación propia.

En el cuadro 2 de este apéndice se presenta la prueba de significancia para el valor de los parámetros estimados en el periodo de análisis previo a la vigencia del programa de 2013 (enero 1980 a diciembre de 2012).

Cuadro 2. Prueba de significancia para el modelo ARIMA $(5,2,0)(2,0,0)$ sin intervención del programa de condonación de contribuciones 2013

\begin{tabular}{|c|c|c|c|c|c|}
\hline \multicolumn{6}{|c|}{ Prueba z para los coeficientes del modelo ARIMA para 2013 sin intervención } \\
\hline & Valor coeficiente & Error estándar & Valor de $\mathrm{z}$ & $\operatorname{Pr}(>|z|)$ & Significancia \\
\hline ar1 & -1.2821 & 0.0482 & -26.5988 & $<2.2 \mathrm{E}-16$ & *** \\
\hline $\operatorname{ar2}$ & -1.2586 & 0.077 & -16.3517 & $<2.2 \mathrm{E}-16$ & $* * *$ \\
\hline ar3 & -0.9309 & 0.0868 & -10.7286 & $<2.2 \mathrm{E}-16$ & $* * *$ \\
\hline $\operatorname{ar} 4$ & -0.5601 & 0.0761 & -7.3575 & $1.88 \mathrm{E}-13$ & $* * *$ \\
\hline ar5 & -0.3254 & 0.0492 & -6.6102 & $3.84 \mathrm{E}-11$ & *** \\
\hline sar1 & 0.3963 & 0.0513 & 7.7194 & $1.17 \mathrm{E}-14$ & $* * *$ \\
\hline sar2 & 0.2325 & 0.0507 & 4.5849 & $4.54 \mathrm{E}-06$ & $* * *$ \\
\hline
\end{tabular}

Fuente: Estimación propia.

En el cuadro 3 de este apéndice se muestra el valor de los parámetros estimados para el programa de condonación de contribuciones federales de 2013, para el periodo de vigencia del programa (enero de 2013).

Cuadro 3. Valor de los parámetros del modelo $\operatorname{ARIMA}(5,2,0)(2,0,0)$ con intervención en enero de 2013

\begin{tabular}{|c|c|c|c|c|c|c|c|c|c|c|}
\hline \multicolumn{10}{|c|}{ Modelo ARIMA } & $(\mathbf{5 , 2 , 0})(\mathbf{2 , 0 , 0}(\mathbf{1 2})$ con intervención \\
\hline & ar1 & ar2 & ar3 & ar4 & ar5 & sar1 & sar2 & Jan13a-MA0 & Jan13b-AR1 & Jan13b-MA0 \\
\hline Coeficientes & -1.2697 & -1.2616 & -0.9562 & -0.5829 & -0.3345 & 0.4242 & 0.2376 & -3.8345 & 0.0086 & 3.8878 \\
\hline Error estándar & 0.0453 & 0.0732 & 0.0824 & 0.0725 & 0.0468 & 0.0476 & 0.0479 & 42.9495 & 0.0932 & 42.9532 \\
\hline
\end{tabular}
Sigma 2 estimado 0.01604: $\log$ likelihood $=281.9$, aic $=-543.81$

Fuente: Estimación propia.

En el cuadro 4 de este apéndice se presenta la prueba de significancia "z" para el valor de los parámetros estimados para el periodo de vigencia del programa de 2013 (enero de 2013).

Cuadro 4. Prueba de significancia para el modelo ARIMA $(5,2,0)(2,0,0)$ con intervención del programa de condonación de contribuciones 2013

\begin{tabular}{|c|c|c|c|c|c|}
\hline \multicolumn{6}{|c|}{ Prueba z para los coeficientes del modelo ARIMA con intervención } \\
\hline & Valor coeficiente & Error estándar & Valor de $\mathrm{z}$ & $\operatorname{Pr}(>|\mathbf{z}|)$ & Significancia \\
\hline ar1 & -1.2697 & 0.0453 & -28.6195 & $<2.2 \mathrm{E}-16$ & $* * *$ \\
\hline ar2 & -1.2616 & 0.0732 & -17.2247 & $<2.2 \mathrm{E}-16$ & $* * *$ \\
\hline ar3 & -0.9562 & 0.0824 & -11.6069 & $<2.2 \mathrm{E}-16$ & $* * *$ \\
\hline ar4 & -0.5829 & 0.0725 & -8.043 & $8.76 \mathrm{E}-16$ & $* * *$ \\
\hline ar5 & -0.3345 & 0.0468 & -7.1507 & $8.63 \mathrm{E}-13$ & $* * *$ \\
\hline sar1 & 0.4242 & 0.0476 & 8.9103 & $<2.2 \mathrm{E}-16$ & $* * *$ \\
\hline sar2 & 0.2376 & 0.0479 & 4.9627 & $6.95 \mathrm{E}-07$ & $* * *$ \\
\hline Jan13a-MA0 & -3.8345 & 42.9495 & -0.0893 & 0.9289 & \\
\hline Jan13b-AR1 & 0.0086 & 0.0932 & 0.0925 & 0.9263 & \\
\hline Jan13b-MA0 & 3.8878 & 42.9532 & 0.0905 & 0.9279 & \\
\hline
\end{tabular}

Significado del código: 0 “***», 0.001 “**”, 0.01 “*”, 0.05 ".” y 0.1 " "

Fuente: Estimación propia. 